\title{
Animal models for COVID-19
}

https://doi.org/10.1038/s41586-020-2787-6

Received: 22 June 2020

Accepted: 15 September 2020

Published online: 23 September 2020

Check for updates

\author{
César Muñoz-Fontela ${ }^{1,2}$, William E. Dowling ${ }^{3}$, Simon G. P. Funnell ${ }^{4}$, Pierre-S. Gsell ${ }^{5}$, \\ A. Ximena Riveros-Balta ${ }^{5}$, Randy A. Albrecht ${ }^{6}$, Hanne Andersen ${ }^{7}$, Ralph S. Baric ${ }^{8}$, \\ Miles W. Carroll ${ }^{4}$, Marco Cavaleri ${ }^{9}$, Chuan Qin ${ }^{10}$, Ian Crozier $^{11}$, Kai Dallmeier $^{12}$, Leon de Waal ${ }^{13}$, \\ Emmie de Wit $^{14}$, Leen Delang ${ }^{12}$, Erik Dohm ${ }^{15}$, W. Paul Duprex ${ }^{16}$, Darryl Falzarano ${ }^{17}$, \\ Courtney L. Finch ${ }^{18}$, Matthew B. Frieman ${ }^{19}$, Barney S. Graham ${ }^{20}$, Lisa E. Gralinski ${ }^{8}$, \\ Kate Guilfoyle ${ }^{13}$, Bart L. Haagmans ${ }^{21}$, Geraldine A. Hamilton ${ }^{22}$, Amy L. Hartman ${ }^{16}$, \\ Sander Herfst ${ }^{21}$, Suzanne J. F. Kaptein ${ }^{12}$, William B. Klimstra ${ }^{23}$, Ivana Knezevic ${ }^{5}$, \\ Philip R. Krause ${ }^{24}$, Jens H. Kuhn ${ }^{18}$, Roger Le Grand ${ }^{25}$, Mark G. Lewis ${ }^{7}$, Wen-Chun Liu ${ }^{6}$, \\ Pauline Maisonnasse ${ }^{25}$, Anita K. McElroy ${ }^{26}$, Vincent Munster ${ }^{14}$, Nadia Oreshkova ${ }^{27}$, \\ Angela L. Rasmussen ${ }^{28}$, Joana Rocha-Pereira ${ }^{12}$, Barry Rockx ${ }^{21}$, Estefanía Rodríguez ${ }^{1,2}$, \\ Thomas F. Rogers ${ }^{29}$, Francisco J. Salguero ${ }^{4}$, Michael Schotsaert ${ }^{6}$, Koert J. Stittelaar ${ }^{13}$, \\ Hendrik Jan Thibaut ${ }^{12}$, Chien-Te Tseng ${ }^{30}$, Júlia Vergara-Alert ${ }^{31}$, Martin Beer ${ }^{32}$, Trevor Brasel $^{30}$, \\ Jasper F. W. Chan ${ }^{33}$, Adolfo García-Sastre ${ }^{6}$, Johan Neyts ${ }^{12}$, Stanley Perlman ${ }^{34}$, \\ Douglas S. Reed ${ }^{23}$, Juergen A. Richt ${ }^{35}$, Chad J. Roy ${ }^{36}$, Joaquim Segalés ${ }^{31,37}$, \\ Seshadri S. Vasan ${ }^{38,39}$, Ana María Henao-Restrepo ${ }^{5 凶}$ \& Dan H. Barouch $^{40 凶}$
}

\begin{abstract}
Severe acute respiratory syndrome coronavirus 2 (SARS-CoV-2) is the aetiological agent of coronavirus disease 2019 (COVID-19), an emerging respiratory infection caused by the introduction of a novel coronavirus into humans late in 2019 (first detected in Hubei province, China). As of 18 September 2020, SARS-CoV-2 has spread to 215 countries, has infected more than 30 million people and has caused more than 950,000 deaths. As humans do not have pre-existing immunity to SARS-CoV-2, there is an urgent need to develop therapeutic agents and vaccines to mitigate the current pandemic and to prevent the re-emergence of COVID-19. In February 2020, the World Health Organization (WHO) assembled an international panel to develop animal models for COVID-19 to accelerate the testing of vaccines and therapeutic agents. Here we summarize the findings to date and provides relevant information for preclinical testing of vaccine candidates and therapeutic agents for COVID-19.
\end{abstract}

Although there are discrepancies in the estimated case-to-fatality ratio of COVID-19 in humans, it is clear that severity is age-stratified and that the case-to-fatality ratio in patients over 65 years of age is probably higher than $1 \%{ }^{1}$. Infection with SARS-CoV- 2 is initially characterized by a range of mild symptoms, including fever, cough, dyspnoea and myalgia $^{2}$. In part, these symptoms are caused by the capacity of SARS-CoV-2 to replicate efficiently in the upper respiratory tract. Although most individuals subsequently resolve the infection, the disease may also progress to severe pneumonia. In severe cases, bilateral lung involvement with ground-glass opacities is the most common finding in computed tomography scanning of the chest. Disease progression can then involve acute respiratory distress syndrome and-in some cases-an inflammatory syndrome that resembles septic shock. Histological examination of the lungs of patients showed bilateral diffuse alveolar damage, pulmonary oedema and formation of hyaline membranes ${ }^{3}$. COVID-19 is also characterized by damage to additional organ systems, associated with coagulopathy and characterized by elevated fibrinogen and D-dimer levels that indicate increased thrombus generation and fibrinolysis ${ }^{4}$. Individuals at a higher risk of developing severe COVID-19 include those with underlying conditions, such as obesity, diabetes, hypertension, chronic respiratory disease and cardiovascular disease ${ }^{1}$.
Through the 'Solidarity' trials, the WHO has launched a global campaign to test therapeutic agents and vaccines on an unprecedented scale $^{5}$. To test these and other potential medical countermeasures, it is imperative to identify animal models for COVID-19 that provide measurable readouts for potential interventions and that use representative virus isolates ${ }^{6}$. To this end, the WHO Research \& Development Blueprint Team established an ad hoc expert working group focused on COVID19 modelling (COM), known as WHO-COM. In this Review, we provide a summary of the current literature on animal models for COVID-19 (Table 1, Supplementary Table 1) that includes studies generated by the WHO-COM group since February 2020, which we hope will serve to facilitate further preclinical analysis of vaccines and therapeutic agents.

\section{Mouse models}

The main impediment to the infection of mouse (Mus musculus) cells with SARS-CoV-2 is the lack of appropriate receptors to initiate viral infection. SARS-CoV-2-as severe acute respiratory syndrome coronavirus (SARS-CoV)-uses the cellular surface protein angiotensin-converting enzyme 2 (ACE2) to bind and enter cells, and mouse ACE2 does not effectively bind the viral spike protein ${ }^{7}$. Several strategies have been developed to solve this problem, as detailed here.

A list of affiliations appears at the end of the paper. 
Table 1 | SARS-CoV-2 infection in humans and in animal models

\begin{tabular}{|c|c|}
\hline Trait & Organism \\
\hline \multicolumn{2}{|l|}{ Virus replication } \\
\hline Upper respiratory tract & $\begin{array}{l}\text { Humans, mice, ferrets, non-human } \\
\text { primates, mink, cats and bats }\end{array}$ \\
\hline Lower respiratory tract & $\begin{array}{l}\text { Humans, mice, hamsters, ferrets and } \\
\text { non-human primates }\end{array}$ \\
\hline Other organs & $\begin{array}{l}\text { Humans (GI tract, CNS and kidney), } \\
\text { hACE2 mice (CNS), hamsters, ferrets and } \\
\text { non-human primates (GI tract) }\end{array}$ \\
\hline \multicolumn{2}{|l|}{ Clinical signs } \\
\hline Fever & Human and ferrets \\
\hline Nasal discharge & Humans and ferrets \\
\hline Laboured breathing & Humans and hamsters \\
\hline \multicolumn{2}{|l|}{ Pneumonia } \\
\hline Bilateral lung involvement & $\begin{array}{l}\text { Humans, hamsters and non-human } \\
\text { primates }\end{array}$ \\
\hline Ground-glass opacities & $\begin{array}{l}\text { Humans, hamsters and non-human } \\
\text { primates }\end{array}$ \\
\hline Focal oedema and inflammation & $\begin{array}{l}\text { Humans, hamsters, ferrets and non-human } \\
\text { primates }\end{array}$ \\
\hline ARDS & Humans \\
\hline Transmission & Humans, hamsters, ferrets, cats and bats \\
\hline \multicolumn{2}{|l|}{ Immunology } \\
\hline Seroconversion & $\begin{array}{l}\text { Humans, mice, hamsters, ferrets, } \\
\text { non-human primates and bats }\end{array}$ \\
\hline Neutralizing antibody titres & $\begin{array}{l}\text { Humans, mice, hamsters, ferrets and } \\
\text { non-human primates }\end{array}$ \\
\hline T cell immunity & $\begin{array}{l}\text { Humans, mice, ferrets and non-human } \\
\text { primates }\end{array}$ \\
\hline Pro-inflammatory cytokines & Humans, mice and non-human primates \\
\hline \multicolumn{2}{|l|}{ Demographics } \\
\hline More severe disease in males & Humans, hamsters \\
\hline $\begin{array}{l}\text { More severe disease in older } \\
\text { individuals }\end{array}$ & $\begin{array}{l}\text { Humans, hamsters and non-human } \\
\text { primates }\end{array}$ \\
\hline
\end{tabular}

\section{Virus adaptation to mouse ACE2}

The spike protein of SARS-CoV-2 can be modified to gain effective binding to mouse ACE2. One strategy to achieve this modification is the sequential passaging of SARS-CoV- 2 in mouse lung tissue ${ }^{8}$. This method is successful because populations of RNA viruses consist of a swarm of closely related viral quasispecies. Rare viruses in the swarm that contain mutations in the spike protein that increase their binding affinity to mouse ACE2 are expected to be selected, owing to their higher levels of replication in mouse lungs. Alternatively, SARS-CoV-2 can be adapted to infect mouse cells by using reverse genetics to modify the receptor-binding domain of the virus so that it can infect mouse cells via the mouse ACE2 protein. Using two approaches, mice have been sensitized for infection but have developed only very mild disease ${ }^{9}$. It is likely that additional efforts aimed at adapting SARS-CoV-2 to mice will result in the outgrowth of additional virus variants that can cause more severe disease. These mice will then be useful for pathogenesis studies, and for studies of antiviral agents and vaccines. One potential caveat is that the mutations in the SARS-CoV-2 spike protein that enhance affinity for the mouse ACE2 receptors are located in the receptor-binding domain, which is the primary target for the neutralizing antibody response. These mutations could thus result in a monoclonal antibody that neutralizes the wild-type virus being falsely considered as non-neutralizing.

\section{Expression of human ACE2 in genetically modified mice}

Another approach to infect mice with SARS-CoV-2 consists of modifying the mice to express human ACE2. There are currently three transgenic mouse models, in which human ACE2 is under the expression of a tissue-specific promoter (for example, the $K r t 18$ promoter for epithelial cells ${ }^{10} ; \mathrm{K} 18$-hACE2 mice), a universal promoter (cytomegalovirus enhancer followed by the chicken $\beta$-actin promoter ${ }^{11}$ ) or the endogenous mouse $A c e 2$ promoter $^{12}$. All of these mice are susceptible to infection by SARS-CoV-2, but differences in their expression of human ACE2 result in a pathogenic range of mild to lethal disease. With the exception of the model in which human ACE2 is controlled by the Ace 2 promoter, mice develop encephalitis after infection with SARS-CoV ${ }^{13}$ or SARS-CoV- $2^{14}$ in these models. However, while SARS-CoV infection of K18-hACE2 mice results in highly lethal encephalitis, the neurological infection caused by SARS-CoV-2 infection in these mice is less severe. Some mice appear to succumb to severe pneumonia, at times at which the brain infection is not substantial ${ }^{15}$. Notably, these mice develop evidence of thrombosis and anosmia after infection with SARS-CoV-2 and have been used for studies of the innate and $\mathrm{T}$ cell responses ${ }^{16}$. Mice develop severe disease after infection with SARS-CoV-2 in these models, and therefore may provide proof-of-concept data to support vaccine and therapeutic efficacy and may be useful for pathogenesis studies.

An alternative approach that mirrors the tissue-specific expression of human ACE2 is to substitute the Ace2 gene with the human ACE2 gene. Similar models that express human dipeptidyl peptidase 4the receptor used by Middle East respiratory syndrome coronavirus (MERS-CoV)-have successfully been developed ${ }^{17-19}$. One mouse model humanized with human ACE2 has been reported, and supports replication of SARS-CoV-2 in respiratory and brain tissues (although mice do not develop severe disease $)^{20}$. However, more severe disease is expected to occur in human ACE2 knock-in mice if virus is passaged serially through mouse lungs. Overall, these mice will probably be very useful models of human disease-especially if combined with viral adaptation that increases the virulence of SARS-CoV- 2 in mice.

Finally, instead of permanent genetic modification, it is also possible to generate mice that are susceptible to infection with SARS-CoV-2 by sensitizing the respiratory tract of these mice to SARS-CoV-2 replication through transduction with adenovirus or adeno-associated virus that expresses human ACE2 (Ad5-hACE2 or AAV-hACE2, respectively). This system, which was pioneered in studies of MERS ${ }^{21}$, allows the transient replication of SARS-CoV-2 in the lungs of mice for several days until immune clearance, and it has the advantage that it can be applied quickly to different strains of mice. Upon infection with SARS-CoV-2, mice transduced with Ad5-hACE2 develop a widespread infection of the lungs and histopathological changes that are consistent with viral pneumonia. These mice developed clinical disease, as characterized by changes in body scoring (hunching) and weight loss. Virus is generally cleared by seven days after infection, although not in some immunocompromised mice ${ }^{14,22}$. Mice sensitized via AAV-hACE2 delivery are also susceptible to infection with SARS-CoV-2, but virus replication seems to be lower than in mice transduced with Ad5-hACE2 ${ }^{23}$. Mice sensitized with Ad5-hACE2 or AAV-hACE2 are useful for evaluating vaccines and antiviral therapies, as well identifying SARS-CoV-2-specific antibody and $T$ cell epitopes. A limitation with these mice-as well as in some of the transgenic mice expressing human ACE2-is that human ACE2 is expressed ectopically, which may change the tissue or cellular tropism of the virus.

\section{Other mouse models and approaches}

Additional ongoing efforts to develop mouse models for studying SARS-CoV-2 infection involve mice humanized with human ACE2 and human haematopoiesis, or Collaborative Cross mice. Severely immunodeficient mice transplanted with human immune cells have widely been used to study human-specific viral infections ${ }^{24,25}$, and the combination 
of human immune system and ACE2 expression could help to further explore the efficacy of vaccines and therapies-in particular, those that modulate human immune cells. Similarly, previous studies have shown that the Collaborative Cross model of genetic diversity (a panel of recombinant inbred mice with expanded susceptibility to viruses that normally do not cause disease in laboratory mice) can be used to enhance virus disease susceptibility; however, infection remains heavily dependent on a functional entry receptor ${ }^{26,27}$. Collaborative Cross mice were previously used with mouse-adapted SARS-CoV to identify mechanisms of pathogenesis and genetic loci that determine susceptibility $^{28}$. Presumably, Collaborative Cross studies could enable the exploration of an expanded range of SARS-CoV-2 phenotypes in mice that potentially better recapitulates human disease, as mouse-adapted strains become available.

In summary, several mouse models of mild and severe COVID-19 have been described or are under development. All of these models will be useful for the evaluation of vaccines and antiviral agents, and some share features with the human disease. At present, no mouse model recapitulates all aspects of COVID-19 in humans, especially the unusual features such as the pulmonary vascular disease and hyperinflammatory syndromes observed in adults and children, respectively ${ }^{29,30}$. However, continued refinement may result in models even for these aspects of the human disease.

\section{Syrian hamster model}

Syrian hamsters (Mesocricetus auratus) are small mammals that have been used as models for infection with respiratory viruses, including SARS-CoV, influenza virus and adenovirus ${ }^{31-34}$. In silico comparison of the ACE2 sequence of humans-known to interact with the receptor-binding domain of theSARS-CoV-2 spike glycoprotein-with that of hamsters ${ }^{35}$ suggested that Syrian hamsters might be susceptible to infection with SARS-CoV-2. Upon experimental intranasal infection, Syrian hamsters show mild-to-moderate disease with progressive weight loss that starts very early after infection (days 1-2 after inoculation). All hamsters that have been challenged by different groups and with different SARS-CoV-2 isolates consistently showed signs of respiratory distress, including laboured breathing ${ }^{35,36}$. Additional signs of morbidity included lethargy, ruffled fur and a hunched posture ${ }^{35}$. After two weeks of infection, hamsters typically recovered. Of particular interest is the fact that infection with SARS-CoV-2 in hamsters reflects some of the demographic differences of COVID-19 in humans. Thus, aged hamsters and male hamsters seem to develop a more severe disease than young and female hamsters, respectively ${ }^{37,38}$.

In hamsters, SARS-CoV-2 infection is associated with high levels of virus replication and histopathological evidence of disease, which included ground-glass opacities and evidence of gas in the cavity surrounding the lungs ${ }^{38}$. These findings are similar to those previously reported for SARS-CoV infection in this model ${ }^{32}$. Viral RNA is readily detected in the respiratory tract and other tissues (such as the small intestine), which could be useful for the evaluation of therapeutic agents and vaccines. Virus transmission to cage-mates has also been observed $^{35}$, which suggests that hamsters may be useful in transmission studies. Histologically, inflammatory infiltrates with abundant expression of viral antigen and apoptosis were observed in the upper and lower respiratory tract, starting at 2 days after infection, being at their most severe at 4 days after infection and resolving at 14 days after infection. Among the non-respiratory-tract tissues, only the intestine demonstrated expression of viral antigen in association with severe epithelial-cell necrosis, damaged and deformed intestinal villi, and increased infiltration of the lamina propria by mononuclear cells. Lung disease was also demonstrated by computed tomography. High-resolution micro-computed tomography scans showed airway dilation and substantial consolidations in the lungs of infected hamsters $^{36}$. A quantitative analysis revealed an increase in the non-aerated lung volume in these hamsters. This method thus allows quantitative monitoring of disease without the need to euthanize the animals.

Expression of chemokines and cytokines in the lungs of hamsters peaked at four days after infection, and then gradually resolved by seven days after infection. Interferon- $\gamma$, and pro-inflammatory chemokines and cytokines, were potently induced at two and four days after infection, respectively, and dropped to the baseline level at seven days after infection. SARS-CoV-2-induced lung pathology in hamsters appears to be driven by immune pathology, as lung injury at four days after infection is markedly reduced in STAT2-knockout hamsters whereas viral loads are massively increased and viral RNA is disseminated in several peripheral tissues ${ }^{36}$. Serum neutralizing antibodies were detected as early as seven days after infection. Passive immunization of naive hamsters with samples of this convalescent serum resulted in significantly reduced viral loads in the respiratory tract, but no obvious improvement in clinical signs and histological changes. Furthermore, SARS-CoV-2 can be transmitted between hamsters via close contact and non-contact routes ${ }^{35,39}$. Transmission via fomites was possible, but not efficient ${ }^{39}$.

Because studies in hamsters can be completed quickly and in a cost-effective manner, there is an increasing interest in the use of this model for screening of therapeutic agents. Limited or no efficacy has been demonstrated for the repurposed drugs hydroxychloroquine (with or without azithromycin) and favipiravir-although high doses of favipiravir did reduce infectious virus titres in the lungs of infected hamsters ${ }^{40,41}$. A YF17D-vectored SARS-CoV-2 vaccine candidate conferred efficient protection against SARS-CoV-2 challenge in hamsters ${ }^{42}$. Adoptive transfer of SARS-CoV-2 neutralizing antibodies protected hamsters from SARS-CoV-2-induced disease ${ }^{43}$. A caveat of hamster models is the lack of research tools for this species-these remain scarce when compared (for example) with those available for mice.

\section{Ferret models}

Ferrets (Mustela putorius furo) have been shown to be a highly valuable model for testing the pathogenicity and transmission of human respiratory viruses, including influenza virus and respiratory syncytial virus $^{44,45}$. It is thus not surprising that the ferret model has been investigated for studies of the pathogenesis of COVID-19 and SARS-CoV-2 transmission. Despite the use of different isolates of SARS-CoV-2, the results have been notably consistent across all laboratories.

Following mucosal exposure to SARS-CoV-2, clinical alterations in ferrets are undetectable or mild and may include lethargy, nasal discharge, wheezing, oropharyngeal build-up of mucus, sneezing and loose stools ${ }^{46}$. Ferrets infected by small-particle aerosols had similar disease, albeit at 100-fold lower doses. Peaks of elevated body temperatures have been observed in some studies, although alterations in body weight are absent or minimal. Minor alterations in haematological parameters, such as mild lymphopenia and neutrophilia, have also been observed. Shedding of SARS-CoV-2 virus is observed in nasal and oropharyngeal swabs ${ }^{77-50}$. As with Syrian hamsters, virus replication is detected in the upper respiratory tract very early after infection (day 2) and is detectable during two weeks of infection. Virus replication in ferrets appears to be restricted to the respiratory and gastrointestinal tracts.

The predominant histopathology findings in SARS-CoV-2-infected ferrets euthanized at the peak of virus replication include inflammation within alveolar spaces and perivascular mononuclear inflammation. In addition, in the larger airways of these ferrets, bronchial submucosal foci with eosinophilic material and collagen fragments (suggestive of collagen degeneration) were observed. Microscopic findings in euthanized ferrets were mild, and included broncho-alveolar or alveolar inflammation.

Ferrets also are able to transmit virus efficiently to uninfected ferrets in experimental settings. Efficient transmission occurred from 


\section{Review}

experimentally infected ferrets to naive cage-mates; transmission from exposed ferrets to companion ferrets that were separated by steel grids did occur, but was not efficient ${ }^{49,51}$. These studies indicated that airborne transmission of SARS-CoV-2 can occur, and suggested that the ferret model may be useful for further transmission studies.

To date, studies performed in ferrets strongly indicate that experimental SARS-CoV-2 infection results in a predominantly upper-respiratory-tract infection in these animals. These findings make the ferret model well-suited to testing the efficacy of mucosal vaccines and therapeutic agents that aim to prevent upper airway infection and/or transmission.

\section{Non-human-primate models}

Non-human-primate models have been explored for COVID-19 in rhesus macaques (Macaca mulatta), cynomolgus macaques (Macaca fascicularis) and African green monkeys (Chlorocebus aethiops). Studies from several laboratories have shown high levels of viral replication for 7-14 days (including both viral RNA and infectious virus) in both the upper and lower respiratory tract, pathological features of viral pneumonia and the variable induction of mild clinical disease ${ }^{52-55}$. Only mild clinical disease has been reported in non-human primates, and insufficient comparable data exist at this time to determine whether there is more clinical disease in rhesus macaques, cynomolgus macaques or African green monkeys. The induction of innate, humoral and cellular immune responses as well as robust protection against rechallenge has also been reported, which demonstrates the induction of natural protective immunity in this model ${ }^{54}$. Non-human primates inoculated via multiroute mucosal, intrabronchial and aerosol exposure showed radiographic abnormalities (by chest X-ray, computed tomography scan or fluorodeoxyglucose positron emission tomography scan) within 2 days, which tended to resolve by 11-15 days after infection. Evidence of the shedding of live virus has been found in both the respiratory and gastrointestinal tracts. In addition, haematological changes - with evidence of $\mathrm{T}$ cell activation, mild lymphopenia and neutrophilia-have been observed in infected non-human primates.

In humans, infection with SARS-CoV-2 in older individuals is associated with an adverse clinical outcome. Currently, two non-human-primate studies in rhesus and cynomolgus macaques have focused on the effect of age on infection with SARS-CoV- $2^{53,56}$. Both studies showed that aged macaques shed virus from nose and throat for longer periods of time that do young adult macaques. Higher viral loads were also detected in lung tissue of aged rhesus macaques. In addition, advanced age in rhesus macaques was also associated with an increased number of radiological and histopathological changes. These studies highlight the importance of including age in the selection criteria of animals, as testing treatment options for severe disease require animal models that recapitulate the disease as seen in humans.

Recent studies have reported the immunogenicity and protective efficacy of several candidates for a COVID-19 vaccine in the rhesus macaque mode $\mathrm{I}^{57-61}$. A concern is that different challenge stocks were used in each of these studies, and may have contributed to the considerable variation in magnitude, consistency and duration of viral replication observed in the control groups in some of these studies. Standardized and sequenced challenge stocks and protocols will be needed to compare vaccine efficacy in non-human primates. Despite this caveat, the vaccines tested so far have induced binding and neutralizing antibodies and have resulted in substantial reductions of viral replication in the lower respiratory tract, and-to a lesser extent-the upper respiratory tract, following challenge with SARS-CoV-2. These findings raise the possibility that vaccines may be more effective at blocking disease of the lower respiratory tract than of the upper respiratory tract. Anamnestic immune responses were observed in some studies, but not others, following challenge, which suggests that protection is often mediated by rapid immunological control but that complete protection may also be possible. Vaccine-elicited neutralizing-antibody titres also correlated with protective efficacy ${ }^{57}$.

\section{Additional animal models}

In addition to animal models that are more commonly used in infectious disease research, recent studies have characterized infection with SARS-CoV-2 in other animals. Here we highlight these recent findings, which may have implications for virus ecology and the evolution of the current pandemic.

\section{Mink}

The mink (Neovison vison), which is a member of the Mustelidae, has previously been shown to be susceptible to infection with SARS-CoV ${ }^{62}$; mink lung epithelial cells and lung-derived cells could also be infected with SARS- $\mathrm{CoV}^{63}$. Mink are also naturally susceptible to infection with SARS-CoV-2. In the Netherlands, an infection of mink with SARS-CoV-2 on two breeding farms was detected at the end of April 2020-most probably as a result of contact with a farm worker who was infected with SARS-CoV- $2^{64}$. At the time of writing, 41 additional mink farms have confirmed infections with SARS-CoV-2 and thousands of mink have been culled in the Netherlands and Spain. In contrast to ferrets, mink displayed moderate respiratory signs that included laboured breathing, and some mink died as a result of infection. SARS-CoV-2 virus was found in the majority of throat and rectal swabs collected from dead mink from both farms. Similar to ferrets, the viral loads in mink were higher in the throat swabs than in the rectal swabs. Although mink may represent a suitable model for moderate-to-severe COVID-19, they are difficult to handle under laboratory conditions.

\section{Cats}

Three experiments have demonstrated that domestic cats (Felis catus) are highly susceptible to infection with SARS-CoV-2 and are able to transmit the virus to naive cats $\mathrm{s}^{50,65,66}$. For example, the inoculation of the SARS-CoV-2 isolate CTan-H into juvenile (70-100 days old) and subadult cats (6-9 months old) through the intranasal route resulted in virus replication in the upper and lower respiratory tract, as well as in the gastrointestinal tract. Both experimentally infected and contact cats seroconverted. At necropsy, interstitial pneumonia, loss of cilia and epithelial necrosis, as well as inflammation in nasal turbinates and trachea, were observed. The authors did not describe clinical signs in any of the infected cats, except that 2 juvenile cats (out of 10 in total) died (on day 3 and day 13 after infection $)^{50}$. Virus antigen was found in epithelial cells of the nasal turbinates, necrotic debris in the tonsil, submucosal glands of the trachea and enterocytes of the small intestine. SARS-CoV-2 transmission by droplets was also demonstrated ${ }^{50}$. Although cats may represent a suitable model for asymptomatic-to-moderate COVID-19, before they are used as such we should be sure that the benefits outweigh the concerns of using companion animals for research; furthermore, cats are difficult to handle in biosafety level-3 containment, and are not a standard animal model. However, owing to their close contact to humans, additional studies-for example, on environmental contamination (cages, beds, food and water bowls, litterboxes and so on) or on transmission efficiency-may be important to inform veterinary and public health authorities about the risk of cats as intermediate hosts or virus carriers at the interface between SARS-CoV-2, humans and animals.

\section{Dogs}

Dogs (Canis lupusfamiliaris) have been shown to be susceptible to SARS-CoV-2, but to a very mild degree. Two experiments have so far been published in this species, which conclude that dogs have a low susceptibility to infection with SARS-CoV- $2^{50,66}$. The susceptibility of both cats and dogs to natural and experimental infection with SARS-CoV-2 strongly suggests that antibody testing in these species could be a 
useful tool for epidemiological studies, in particular in areas with high density of cases of COVID-19 in humans.

\section{Pigs}

In silico data suggested that swine ACE2 should bind the spike protein of SARS-CoV-2. However, several experimental infections performed in pigs (Sus scrofa domesticus) by different research groups indicate that this species is not susceptible to infection with SARS-CoV-2 in vivo ${ }^{49,50}$. No clinical signs and no clear evidence of virus replication have been observed in pigs. Therefore, pigs do not appear to represent a suitable animal model for COVID-19. Conversely, previous studies have reported infection with SARS-CoV in pigs ${ }^{67}$. Experimental infection of pigs with SARS-CoV resulted in the detection of viral RNA in the blood and seroconversion, but not in clinical signs or virus isolation, which ruled out pigs as amplifying hosts for SARS-CoV ${ }^{68}$. By contrast, infection with another bat betacoronavirus - known as swine acute diarrhoea syndrome coronavirus (SADS-CoV)- has been demonstrated in swine ${ }^{69}$. Therefore, owing to their importance as livestock and the enormous global number of pigs, it may be important for future studies to address the putative susceptibility of additional pig breeds to infection with SARS-CoV-2.

\section{Chickens and ducks}

At least one in silico study using the informational spectrum methodology proposed chicken as an animal species that is potential susceptible to infection with SARS-CoV- $2^{70}$. However, the limited experimental studies performed so far have suggested that chickens-including embryonated chicken eggs-and ducks are not susceptible to infection with SARS-CoV-2 $2^{49,50,71}$. Neither chickens nor ducks appear to represent suitable animal models for studies of SARS-CoV-2 infection. These findings are similar to those previously reported for infection with SARS-CoV, in which experimental inoculation of different bird species with SARS-CoV (including chickens) resulted in neither replication nor seroconversion $^{72}$.

\section{Fruit bats}

Pre-pandemic studies that assessed the potential emergence of SARS-like coronaviruses in bats indicated that some of these viruses were able to use several orthologues of human ACE2 for docking and entry ${ }^{73,74}$. These studies underscored the importance of coronavirus surveillance studies in bats, as these animals are regarded as the natural reservoir of many coronaviruses-including SARS-CoV and SARS-CoV- $2^{75,76}$. The intranasal inoculation of fruit bats (Rousettus aegyptiacus) with SARS-CoV-2 resulted in efficient replication in the upper respiratory tract and seroconversion in seven out of nine of the bats. Transmission occurred to one out of three direct-contact animals. Clinical signs were absent, but rhinitis could be detected by immunohistology ${ }^{49}$. Conversely, previous studies showed that a SARS-like coronavirus did not replicate in fruit bats after experimental inoculation ${ }^{77}$. These findings suggest that, although Rousettus bats are not the original reservoir species of SARS-CoV-2, experimental infection of these fruit bats could help to model the physiopathology of the virus in its host.

\section{Preclinical alternatives to animal models}

Historically, animal alternatives for studying respiratory viruses have involved in vitro approaches such as cell lines (for example, Vero, A549 and MDCK cell lines) or primary-tissue-derived human cells in conventional cell culture. However, over the past decade, advances in engineering, cell biology and microfabrication have come together to enable the development of new human-cell-based alternatives to animal models. In this regard, micro-engineered organs-on-chips and lung organoids have been shown to support key hallmarks of the cytopathology and inflammatory responses observed in human airways after infection with SARS-CoV-2 and have served to facilitate the study of human disease pathogenesis, test candidate COVID-19 therapeutic agents and expedite drug repurposing ${ }^{78,79}$.

\section{Perspectives}

Since SARS-CoV-2 emerged in the human population in late 2019 , it has spread via human-to-human transmission to most countries in the world, leading to a coronavirus pandemic of an unprecedented scale. Under the umbrella of the WHO, the WHO-COM is fostering the development of animal models for COVID-19 through international exchange of protocols, unpublished data and ideas across many laboratories in the world. As discussed in this Review, a number of studies have been conducted-many of them by members of the WHO-COM-that indicate that some of the animal models support viral replication.

A study ${ }^{80}$ based on the three-dimensional X-ray structure of SARS-CoV-2 spike protein bound to human ACE2 has discussed the variance observed between 19 different animal species, as well as within 3 colonies of the same species of bat from different provinces within China. This analysis noted that many predicted affinities of the spike protein for the ACE2 receptor (especially those of dog and pig) did not match the relative natural resistance of the corresponding species to SARS-CoV-2. This was proposed to be due to differences between species in the levels of ACE2 expression in the respiratory epithelium ${ }^{80}$. Similarly, a recent study aimed to predict the host range of SARS-CoV-2 through a comparative structural analysis of ACE2 in more than 400 vertebrates. These data show discrepancies between the predicted susceptibilities to infection and those experimentally observed; ferrets, for example, were predicted to have a very low susceptibility to infection ${ }^{81}$. These data suggest that susceptibility to infection may be a function of several factors, including genetic $A C E 2$ composition, organ-specific ACE2 expression and other host factors (such as additional receptors and host immune responses).

One immediate goal of the WHO-COM group is to evaluate whether mimicking human comorbidities, co-infections or the immune senescence associated with age in animal models may result in more-severe disease phenotypes. The existing animal models have also been valuable for testing vaccines and therapeutic agents. Several vaccine candidates have shown protection in rhesus macaques ${ }^{57-61}$, and both the cynomolgus and rhesus macaque models have been useful for the testing of therapeutic agents ${ }^{82}$. In future studies, it will be important to define key outcome measures that would allow comparison between candidate interventions in animal models and humans. Many of the pathogenesis studies described in this Review have also highlighted an important caveat in COVID-19 research, which are the methods used to measure virus replication. The group found that viral RNA or genome copy numbers measured by quantitative PCR assays were three-to-four orders of magnitude higher than infectious virus titres measured by cell culture assays, and thus combining cytopathogenic effect or plaque assays with the PCR-based quantification of viral RNA is a prudent approach to evaluating virus shedding and the potential for virus transmission. Standardization of these measurements will be important for the future evaluation of vaccines and therapeutic agents.

There have been concerns that coronaviruses might pose a risk of vaccine-associated enhanced respiratory disease or antibody-dependent enhancement of virus entry and replication in cells bearing the Fc receptor ${ }^{83}$. These types of syndrome have been linked to vaccines that induced substantial levels of non-neutralizing antibodies or responses biased toward type-2 helper CD4 T cells. Therefore, evaluating the relative potency of neutralizing activity to overall binding antibody and obtaining evidence for responses biased toward the CD4 subset of T cells through cytokine production or antibody-subtype response patterns would be informative. To ensure such models are able to provide these readouts, it is important to attempt to induce vaccine-associated enhanced respiratory disease in models of COVID-19 challenge using suboptimal doses of candidate vaccines or antigenic 


\section{Review}

preparations with the goal of inducing the required detrimental immune profile and associated lung pathology.

\section{Outlook}

There are a number of small and large animal models that investigators can use to explore important aspects of COVID-19, including pathology, transmission and host responses to SARS-CoV-2, as well as to help to establish the safety and efficacy of potential therapeutic agents and vaccines. Future studies will need to standardize challenge stocks, assays and protocols to allow comparisons of different candidate interventions. Animal models are needed to assess vaccine-associated enhanced respiratory disease, and the establishment of a positive control for this disease will be important.

Continued refinement and development of animal models for COVID19 will contribute to the development of vaccines, therapeutic agents and other countermeasures. Large-scale clinical trials are currently underway to test multiple candidate preventative and therapeutic interventions in humans. The outcomes of these clinical-efficacy trials will allow an unprecedented opportunity for the back-validation and refinement of these animal models.

1. Verity, R. et al. Estimates of the severity of coronavirus disease 2019: a model-based analysis. Lancet Infect. Dis. 20, 669-677 (2020).

2. Tang, D., Comish, P. \& Kang, R. The hallmarks of COVID-19 disease. PLoS Pathog. 16, e1008536 (2020).

3. Chau, V. Q. et al. The imperfect cytokine storm: severe COVID-19 with ARDS in a patient on durable LVAD support. JACC: Case Reports 2, 1315-1320 (2020).

4. Ji, H.-L., Zhao, R., Matalon, S. \& Matthay, M. A. Elevated plasmin(ogen) as a common risk factor for COVID-19 susceptibility. Physiol. Rev. 100, 1065-1075 (2020).

5. Hodgson, J. The pandemic pipeline. Nat. Biotechnol. 38, 523-532 (2020).

6. Bauer, D. C. et al. Supporting pandemic response using genomics and bioinformatics: a case study on the emergent SARS-CoV-2 outbreak. Transbound. Emerg. Dis. 67, 1453-1462 (2020).

7. Wan, Y., Shang, J., Graham, R., Baric, R. S. \& Li, F. Receptor recognition by novel coronavirus from Wuhan: an analysis based on decade-long structural studies of SARS coronavirus. J. Virol. 94, e00127-20 (2020)

8. Gu, H. et al. Rapid adaptation of SARS-CoV-2 in BALB/c mice: novel mouse model for vaccine efficacy. Preprint at https://doi.org/10.1101/2020.05.02.073411 (2020).

9. Dinnon, K. H. et al. A mouse-adapted model of SARS-CoV-2 to test COVID-19 countermeasures. Nature https://doi.org/10.1038/s41586-020-2708-8 (2020)

10. McCray, P. B. Jr et al. Lethal infection of K18-hACE2 mice infected with severe acute respiratory syndrome coronavirus. J. Virol. 81, 813-821 (2007).

11. Tseng, C.-T. K. et al. Severe acute respiratory syndrome coronavirus infection of mice transgenic for the human angiotensin-converting enzyme 2 virus receptor. J. Virol. 81, 1162-1173 (2007)

12. Bao, L. et al. The pathogenicity of SARS-CoV-2 in hACE2 transgenic mice. Nature 583, 830-833 (2020)

A study that shows that transgenic mice that express human ACE2 undergo severe disease after SARS-CoV-2 infection.

13. Netland, J., Meyerholz, D. K., Moore, S., Cassell, M. \& Perlman, S. Severe acute respiratory syndrome coronavirus infection causes neuronal death in the absence of encephalitis in mice transgenic for human ACE2. J. Virol. 82, 7264-7275 (2008).

14. Rathnasinghe, R. et al. Comparison of transgenic and adenovirus hACE2 mouse models for SARS-CoV-2 infection. Preprint at https://doi.org/10.1101/2020.07.06.190066 (2020).

15. Winkler, E. S. et al. SARS-CoV-2 infection in the lungs of human ACE2 transgenic mice causes severe inflammation, immune cell infiltration, and compromised respiratory function. Preprint at https://doi.org/10.1101/2020.07.09.196188 (2020).

16. Zheng, J. et al. K18-hACE2 mice for studies of COVID-19 treatments and pathogenesis including anosmia. Preprint at https://doi.org/10.1101/2020.08.07.242073 (2020).

17. Cockrell, A. S. et al. A mouse model for MERS coronavirus-induced acute respiratory distress syndrome. Nat. Microbiol. 2, 16226 (2017).

18. Li, K. et al. Mouse-adapted MERS coronavirus causes lethal lung disease in human DPP4 knockin mice. Proc. Natl Acad. Sci. USA 114, E3119-E3128 (2017).

19. Pascal, K. E. et al. Pre- and postexposure efficacy of fully human antibodies against Spike protein in a novel humanized mouse model of MERS-CoV infection. Proc. Natl Acad. Sci. USA 112, 8738-8743 (2015).

20. Sun, S.-H. et al. A mouse model of SARS-CoV-2 infection and pathogenesis. Cell Host Microbe 28, 124-133.e4 (2020).

21. Zhao, J. et al. Rapid generation of a mouse model for Middle East respiratory syndrome. Proc. Natl Acad. Sci. USA 111, 4970-4975 (2014).

22. Hassan, A. O. et al. A SARS-CoV-2 infection model in mice demonstrates protection by neutralizing antibodies. Cell 182, 744-753.e4 (2020).

23. Israelow, B. et al. Mouse model of SARS-CoV-2 reveals inflammatory role of type I interferon signaling. J. Exp. Med. 217, e20201241 (2020).

24. Spengler, J. R. et al. Severity of disease in humanized mice infected with Ebola virus or Reston virus is associated with magnitude of early viral replication in liver. J. Infect. Dis. 217, 58-63 (2018).

25. Frias-Staheli, N. et al. Utility of humanized BLT mice for analysis of dengue virus infection and antiviral drug testing. J. Virol. 88, 2205-2218 (2014).
26. Price, A. et al. Transcriptional correlates of tolerance and lethality in mice predict Ebola virus disease patient outcomes. Cell Rep. 30, 1702-1713.e6 (2020).

27. Rasmussen, A. L. et al. Host genetic diversity enables Ebola hemorrhagic fever pathogenesis and resistance. Science 346, 987-991 (2014).

28. Gralinski, L. E. et al. Genome wide identification of SARS-CoV susceptibility loci using the collaborative cross. PLoS Genet. 11, e1005504 (2015).

29. Feldstein, L. R. et al. Multisystem inflammatory syndrome in U.S. children and adolescents. N. Engl. J. Med. 383, 334-346 (2020).

30. Ackermann, M. et al. Pulmonary vascular endothelialitis, thrombosis, and angiogenesis in Covid-19. N. Engl. J. Med. 383, 120-128 (2020).

31. Miao, J., Chard, L. S., Wang, Z. \& Wang, Y. Syrian hamster as an animal model for the study on infectious diseases. Front. Immunol. 10, 2329 (2019).

32. Roberts, A. et al. Severe acute respiratory syndrome coronavirus infection of golden Syrian hamsters. J. Virol. 79, 503-511 (2005).

33. Iwatsuki-Horimoto, K. et al. Syrian hamster as an animal model for the study of human influenza virus infection. J. Virol. 92, e01693-17 (2018).

34. Wold, W. S. M. \& Toth, K. Chapter three - Syrian hamster as an animal model to study oncolytic adenoviruses and to evaluate the efficacy of antiviral compounds. Adv. Cancer Res. 115, 69-92 (2012).

35. Chan, J. F.-W. et al. Simulation of the clinical and pathological manifestations of coronavirus disease 2019 (COVID-19) in golden Syrian hamster model: implications for disease pathogenesis and transmissibility. Clin. Infect. Dis. ciaa325 (2020).

36. Boudewijns, R. et al. STAT2 signaling as double-edged sword restricting viral dissemination but driving severe pneumonia in SARS-CoV-2 infected hamsters. Preprint at https://doi.org/10.1101/2020.04.23.056838 (2020)

37. Osterrieder, N. et al. Age-dependent progression of SARS-CoV-2 infection in Syrian hamsters. Viruses 12, 779 (2020).

38. Imai, M. et al. Syrian hamsters as a small animal model for SARS-CoV-2 infection and countermeasure development. Proc. Natl Acad. Sci. USA 117, 16587-16595 (2020).

39. Sia, S. F. et al. Pathogenesis and transmission of SARS-CoV-2 in golden hamsters. Nature 583, 834-838 (2020)

A comprehensive description of SARS-CoV-2 pathogenesis in the Syrian hamster model and its applicability for transmission studies.

40. Kaptein, S. J. et al. Antiviral treatment of SARS-CoV-2-infected hamsters reveals a weak effect of favipiravir and a complete lack of effect for hydroxychloroquine. Preprint at https://doi.org/10.1101/2020.06.19.159053 (2020)

41. Driouich, J.-S. et al. Favipiravir and severe acute respiratory syndrome coronavirus 2 in hamster model. Preprint at https://doi.org/10.1101/2020.07.07.191775 (2020).

42. Sanchez-Felipe, L. et al. A single-dose live-attenuated YF17D-vectored SARS-CoV2 vaccine candidate. Preprint at https://doi.org/10.1101/2020.07.08.193045 (2020).

43. Rogers, T. F. et al. Isolation of potent SARS-CoV-2 neutralizing antibodies and protection from disease in a small animal model. Science 369, 956-963 (2020).

44. Enkirch, T. \& von Messling, V. Ferret models of viral pathogenesis. Virology 479-480, 259-270 (2015).

45. Callaway, E. Labs rush to study coronavirus in transgenic animals - some are in short supply. Nature 579, $183(2020)$

46. Blanco-Melo, D. et al. Imbalanced host response to SARS-CoV-2 drives development of COVID-19. Cell 181, 1036-1045.e9 (2020).

A comparative pathogenesis study that compares host immune responses and pathophysiology hallmarks of SARS-CoV-2 infections in several animal models.

47. Kim, Y.-I. et al. Infection and rapid transmission of SARS-CoV-2 in ferrets. Cell Host Microbe 27, 704-709.e2 (2020).

48. Ryan, K. A. et al. Dose-dependent response to infection with SARS-CoV-2 in the ferret model: evidence of protection to re-challenge. Preprint at https://doi.org/10.1101/ 2020.05.29.123810 (2020).

49. Schlottau, K. et al. Experimental transmission studies of SARS-CoV-2 in fruit bats, ferrets, pigs and chickens. Preprint at https://ssrn.com/abstract=3578792 (2020).

50. Shi, J. et al. Susceptibility of ferrets, cats, dogs, and other domesticated animals to SARS-coronavirus 2. Science 368, 1016-1020 (2020). The infection profile of SARS-CoV-2 in ferrets as well as other species, with implications for public health

51. Richard, M. et al. SARS-CoV-2 is transmitted via contact and via the air between ferrets. Nat. Commun. 11, 3496 (2020).

52. Munster, V. J. et al. Respiratory disease in rhesus macaques inoculated with SARS-CoV-2 Nature 585, 268-272 (2020).

The pathogenesis of SARS-CoV-2 infection in the rhesus macaque model.

53. Rockx, B. et al. Comparative pathogenesis of COVID-19, MERS, and SARS in a nonhuman primate model. Science 368, 1012-1015 (2020).

The comparative pathogenesis of SARS-CoV-2 and other highly pathogenic coronaviruses in the non-human primate model.

54. Chandrashekar, A. et al. SARS-CoV-2 infection protects against rechallenge in rhesus macaques. Science 369, 812-817 (2020).

This study provides evidence that natural infection protects against SARS-CoV-2 rechallenge in non-human primates.

55. Finch, C. L. et al. Characteristic and quantifiable COVID-19-like abnormalities in CT- and PET/CT-imaged lungs of SARS-CoV-2-infected crab-eating macaques (Macaca fascicularis). Preprint at https://doi.org/10.1101/2020.05.14.096727 (2020).

56. Yu, P. et al. Age-related rhesus macaque models of COVID-19. Animal Model. Exp. Med. 3, 93-97 (2020)

57. $\mathrm{Yu}$, J. et al. DNA vaccine protection against SARS-CoV-2 in rhesus macaques. Science 369, 806-811 (2020)

58. Gao, Q. et al. Development of an inactivated vaccine candidate for SARS-CoV-2. Science 369, 77-81 (2020)

59. Mercado, N. B. et al. Single-shot Ad26 vaccine protects against SARS-CoV-2 in rhesus macaques. Nature https://doi.org/10.1038/s41586-020-2607-z (2020).

60. van Doremalen, $\mathrm{N}$. et al. ChAdOx $1 \mathrm{nCoV}-19$ vaccine prevents SARS-CoV-2 pneumonia in rhesus macaques. Nature https://doi.org/10.1038/s41586-020-2608-y (2020). 
61. Corbett, K. S. et al. Evaluation of the mRNA-1273 vaccine against SARS-CoV-2 in nonhuman primates. N. Engl. J. Med. https://doi.org/10.1056/NEJMoa2024671 (2020).

62. Shi, Z. \& Hu, Z. A review of studies on animal reservoirs of the SARS coronavirus. Virus Res. 133, 74-87 (2008).

63. Gillim-Ross, L. et al. Discovery of novel human and animal cells infected by the severe acute respiratory syndrome coronavirus by replication-specific multiplex reverse transcription-PCR. J. Clin. Microbiol. 42, 3196-3206 (2004).

64. Oreshkova, N. et al. SARS-CoV-2 infection in farmed minks, the Netherlands, April and May 2020. Euro Surveill. 25, 1016 (2020).

65. Halfmann, P. J. et al. Transmission of SARS-CoV-2 in domestic cats. N. Engl. J. Med. 383, 592-594 (2020).

66. Bosco-Lauth, A. M. et al. Pathogenesis, transmission and response to re-exposure of SARS-CoV-2 in domestic cats. Preprint at https://doi.org/10.1101/2020.05.28.120998 (2020).

67. Chen, W. et al. SARS-associated coronavirus transmitted from human to pig. Emerg. Infect. Dis. 11, 446-448 (2005).

68. Weingartl, H. M. et al. Susceptibility of pigs and chickens to SARS coronavirus. Emerg. Infect. Dis. 10, 179-184 (2004).

69. Zhou, P. et al. Fatal swine acute diarrhoea syndrome caused by an HKU2-related coronavirus of bat origin. Nature 556, 255-258 (2018).

70. Veljkovic, V., Vergara-Alert, J., Segalés, J. \& Paessler, S. Use of the informational spectrum methodology for rapid biological analysis of the novel coronavirus 2019-nCoV: prediction of potential receptor, natural reservoir, tropism and therapeutic/vaccine target. F1000Res. 9, 52 (2020).

71. Barr, I. G., Rynehart, C., Whitney, P. \& Druce, J. SARS-CoV-2 does not replicate in embryonated hen's eggs or in MDCK cell lines. Euro Surveill. 25, 737 (2020).

72. Swayne, D. E. et al. Domestic poultry and SARS coronavirus, southern China. Emerg. Infect. Dis. 10, 914-916 (2004).

73. Menachery, V. D. et al. A SARS-like cluster of circulating bat coronaviruses shows potential for human emergence. Nat. Med. 21, 1508-1513 (2015).

74. Menachery, V. D. et al. SARS-like WIV1-CoV poised for human emergence. Proc. Natl Acad. Sci. USA 113, 3048-3053 (2016).

75. Li, W. et al. Bats are natural reservoirs of SARS-like coronaviruses. Science 310, 676-679 (2005)

76. Andersen, K. G., Rambaut, A., Lipkin, W. I., Holmes, E. C. \& Garry, R. F. The proximal origin of SARS-CoV-2. Nat. Med. 26, 450-452 (2020).

77. van Doremalen, N. et al. SARS-like coronavirus WIV1-CoV does not replicate in Egyptian fruit bats (Rousettus aegyptiacus). Viruses 10, 727 (2018).

78. Han, Y. et al. Identification of candidate COVID-19 therapeutics using hPSC-derived lung organoids. Preprint at https://doi.org/10.1101/2020.05.05.079095 (2020).

79. Si, L. et al. Human organs-on-chips as tools for repurposing approved drugs as potential influenza and COVID19 therapeutics in viral pandemics. Preprint at https://doi.org/ 10.1101/2020.04.13.039917 (2020).

80. Zhai, X. et al. Comparison of severe acute respiratory syndrome coronavirus 2 spike protein binding to ACE2 receptors from human, pets, farm animals, and putative intermediate hosts. J. Virol. 94, 1199 (2020).

81. Damas, J. et al. Broad host range of SARS-CoV-2 predicted by comparative and structural analysis of ACE2 in vertebrates. Proc. Natl Acad. Sci. USA 117, 22311-22322 (2020)

82. Williamson, B. N. et al. Clinical benefit of remdesivir in rhesus macaques infected with SARS-CoV-2. Nature 585, 273-276 (2020).

83. Graham, B. S. Rapid COVID-19 vaccine development. Science 368, 945-946 (2020).

Acknowledgements The content of this Review represents the opinions of the authors, and does not reflect the views or policies of any of their corresponding institutions.

Author contributions All authors contributed to writing this Review.

Competing interests The authors declare no competing interests.

Additional information

Supplementary information is available for this paper at https://doi.org/10.1038/s41586-0202787-6.

Correspondence and requests for materials should be addressed to A.M.H.-R. or D.H.B.
Peer review information Nature thanks Linda Saif and the other, anonymous, reviewer(s) for their contribution to the peer review of this work.

Reprints and permissions information is available at http://www.nature.com/reprints. Publisher's note Springer Nature remains neutral with regard to jurisdictional claims in published maps and institutional affiliations.

(c) Springer Nature Limited 2020

${ }^{1}$ Bernhard Nocht Institute for Tropical Medicine, Hamburg, Germany. ${ }^{2}$ German Center for Infection Research (DZIF), Partner site Hamburg-Lübeck-Borstel-Riems, Hamburg, Germany. ${ }^{3}$ Centre for Epidemic Preparedness Innovations (CEPI), Washington, DC, USA. ${ }^{4}$ National Infection Service, Public Health England, Salisbury, UK. ${ }^{5}$ World Health Organization, Geneva, Switzerland. ${ }^{6}$ Department of Microbiology, Global Health and Emerging Pathogens Institute, Icahn School of Medicine at Mount Sinai, New York, NY, USA. ${ }^{7}$ Bioqual Inc, Rockville, MD, USA. ${ }^{8}$ Department of Epidemiology, University of North Carolina at Chapel Hill, Chapel Hill, NC, USA. ${ }^{9}$ European Medicines Agency, Amsterdam, The Netherlands. ${ }^{10}$ Institute of Laboratory Animal Sciences, Chinese Academy of Medical Sciences and Peking Union Medical College, Peking, China. ${ }^{11}$ Clinical Monitoring Research Program Directorate, Frederick National Laboratory for Cancer Research, Frederick, MD, USA. ${ }^{12} \mathrm{KU}$ Leuven Department of Microbiology, Immunology and Transplantation, Rega Institute, Laboratory of Virology and Chemotherapy, Leuven, Belgium. ${ }^{13}$ Viroclinics Xplore, Schaijk, The Netherlands. ${ }^{14}$ Laboratory of Virology, National Institute of Allergy and Infectious Diseases, National Institutes of Health, Hamilton, MT, USA. ${ }^{15}$ Animal Resources Program, University of Alabama at Birmingham, Birmingham, AL, USA. ${ }^{16}$ Department of Microbiology and Molecular Genetics, Center for Vaccine Research, University of Pittsburgh, Pittsburgh, PA, USA. ${ }^{17}$ VIDO-Intervac, University of Saskatchewan, Saskatoon, Saskatchewan, Canada. ${ }^{18}$ Integrated Research Facility at Fort Detrick, National Institute of Allergy and Infectious Diseases, National Institutes of Health, Frederick, MD, USA. ${ }^{19}$ Department of Microbiology and Immunology, University of Maryland School of Medicine, Baltimore, MD, USA. ${ }^{20}$ Vaccine Research Center, National Institute of Allergy and Infectious Diseases, National Institutes of Health, Bethesda, MD, USA. ${ }^{21}$ Department of Viroscience, Erasmus University Medical Center, Rotterdam, The Netherlands. ${ }^{22}$ Emulate Inc, Boston, MA, USA. ${ }^{23}$ Department of Immunology, Center for Vaccine Research, University of Pittsburgh, Pittsburgh, PA, USA. ${ }^{24}$ Center for Biologics Evaluation and Research, FDA, Silver Spring, MD, USA. ${ }^{25}$ Center for Immunology of Viral, Auto-immune, Hematological and Bacterial diseases (IMVA-HB/IDMIT), Inserm, CEA, Université Paris-Saclay, Paris, France. ${ }^{26}$ Division of Pediatric Infectious Diseases, Center for Vaccine Research, University of Pittsburgh School of Medicine, Pittsburgh, PA, USA. ${ }^{27}$ Wageningen Bioveterinary Research (WBVR), Wageningen University and Research, Lelystad, The Netherlands. ${ }^{28}$ Center for Infection and Immunity, Columbia Mailman ISchool of Public Health, New York, NY, USA. ${ }^{29}$ Division of Infectious Diseases, University of California San Diego, San Diego, CA, USA. ${ }^{30}$ Department of Microbiology and Immunology, University of Texas Medical Branch, Galveston, TX, USA. ${ }^{31}$ Centre de Recerca en Sanitat Animal (CReSA, IRTA-UAB), Campus Universitat Autònoma de Barcelona, Bellaterra, Spain. ${ }^{32}$ Institute of Diagnostic Virology, Friedrich-Loeffler-Institut, Greifswald-Insel Riems, Germany. ${ }^{33}$ Carol Yu Centre for Infection, Department of Microbiology, Li Ka Shing Faculty of Medicine, The University of Hong Kong, Hong Kong, China. ${ }^{34}$ Department of Microbiology and Immunology, University of lowa, lowa City, IA, USA. ${ }^{35}$ College of Veterinary Medicine, Kansas State University, Manhattan, KS, USA. ${ }^{36}$ Tulane National Primate Research Center, Covington, LA, USA. ${ }^{37}$ Departament de Sanitat i Anatomia Animals, Facultat de Veterinària, UAB, Bellaterra, Spain. ${ }^{38}$ Australian Centre for Disease Preparedness, CSIRO, Geelong, Victoria, Australia. ${ }^{39}$ Department of Health Sciences, University of York, York, UK. ${ }^{40}$ Center for Virology and Vaccine Research, Beth Israel Deaconess Medical Center, Harvard Medical School, Boston, MA, USA. ${ }^{\bowtie}$ e-mail: henaorestrepoa@who.int; dbarouch@ bidmc.harvard.edu 